\title{
Effects of Trilostane on urinary Catecholamines and their metabolites in dogs with Hypercortisolism
}

\author{
Nadja Sieber-Ruckstuhl ${ }^{1 *}$ (D) Elena Salesov ${ }^{1}$, Saskia Quante ${ }^{1,4}$, Barbara Riond $^{2}$, Katharina Rentsch ${ }^{3}$, \\ Regina Hofmann-Lehmann ${ }^{2}$, Claudia Reusch ${ }^{1}$ and Felicitas Boretti ${ }^{1}$
}

\begin{abstract}
Background: Glucocorticoids influence the synthesis and metabolism of catecholamines (epinephrine and norepinephrine) and metanephrines (metanephrine and normetanephrine). The aim of this study was to measure urinary catecholamines and metanephrines in dogs with hypercortisolism before and during trilostane therapy.

Urine samples were collected during initial work up and during therapy with trilostane in 14 dogs with hypercortisolism and in 25 healthy dogs. Epinephrine, norepinephrine, metanephrine and normetanephrine were measured using high-pressure liquid chromatography and expressed as ratios to urinary creatinine concentration.

Results: Untreated dogs with hypercortisolism had significantly higher epinephrine, norepinephrine, and normetanephrine: creatinine ratios compared to healthy dogs. During trilostane therapy, urinary catecholamines and their metabolites did not decrease significantly. However, dogs with low post-ACTH cortisol concentrations during trilostane therapy had less increased epinephrine, norepinephrine and normetanephrine:creatinine ratios compared to healthy dogs. There was no correlation of urinary catecholamines and their metabolites with baseline or post-ACTH cortisol or endogenous ACTH concentrations during trilostane therapy.

Conclusion: Influences between steroid hormones and catecholamines seem to occur, as dogs with hypercortisolism have significantly higher urinary epinephrine, norepinephrine, and normetanephrine:creatinine ratios. Once-daily trilostane therapy does not lead to a significant decrease in catecholamines and their metabolites. Trilostane-treated dogs still have increased urinary epinephrine, norepinephrine and normetanephrine:creatinine ratios during trilostane therapy.
\end{abstract}

Keywords: ACTH, Metanephrines, Pheochromocytoma, Trilostane therapy, Canine

\section{Background}

Catecholamines (epinephrine, norepinephrine) are produced by the chromaffin cells of the adrenal medulla and the postganglionic fibers of the sympathetic nervous system. They are synthesized from the amino acid tyrosine, which is converted to L-dihydroxyphenylalanine (L-DOPA), next to dopamine and finally to norepinephrine [1]. In most sympathetic postganglionic neurons, norepinephrine is the final product. In the cells of the adrenal medulla, norepinephrine is further converted into epinephrine [1]. After release into the plasma, catecholamines are quickly

\footnotetext{
*Correspondence: nsieber@vetclinics.uzh.ch

${ }^{1}$ Clinic for Small Animal Internal Medicine, Vetsuisse Faculty University of

Zurich, Zurich, Switzerland

Full list of author information is available at the end of the article
}

metabolized. Metabolism takes place mainly in the adrenal gland cells and only a small proportion in the liver and kidneys and includes the conversion of epinephrine to metanephrine and norepinephrine to normetanephrine [2]. While catecholamines are released intermittently, metanephrines (metanephrine and normetanephrine) are constantly released into the circulation and excreted in the urine [2]. This constant liberation explains why measurement of urinary or plasma metanephrines has a higher accuracy to diagnose pheochromocytoma than the measurement of catecholamines [2].

The adrenal medulla comprises about one-fourth of the adrenal mass. The rest of the adrenal mass consists of the adrenal cortex containing steroid-producing adrenocortical cells. For a long time, the two cell populations 
within the adrenal gland were considered as two independent endocrine systems. However, nowadays, interactions between steroid hormones and catecholamines are well known. Glucocorticoids have been shown to influence several enzymes involved in catecholamine synthesis and metabolism: first, tyrosine hydroxylase, which is the rate-limiting enzyme in catecholamine biosynthesis; second, PNMT (Phenylethanolamin-N-Methyltransferase), which converts norepinephrine to epinephrine; and third, dopamine $\beta$-hydroxylase, which hydroxylates dopamine to norepinephrine [3-7]. In vitro, glucocorticoids have been shown to increase the release of catecholamines from canine adrenal glands [8]. Therefore, it was not surprising that dogs with hypercortisolism $(\mathrm{HC})$ were found to have increased concentrations of urinary epinephrine-, norepinephrine- and normetanephrine-tocreatinine ratios compared to healthy dogs [9]. However, the evolution of urinary catecholamines and metanephrines concentrations during the therapy of canine $\mathrm{HC}$ is unknown. Hypercortisolism is an important differential diagnosis in dogs with pheochromocytomas, as clinical signs of both diseases overlap and pheochromocytomas cannot be distinguished ultrasonographically from tumors of the adrenal cortex.

Therefore, the objective of this study was to evaluate urine concentrations of catecholamines and metanephrines in dogs with $\mathrm{HC}$ before and during therapy with trilostane. We hypothesized that successful therapy of $\mathrm{HC}$ with trilostane would lead to a significant decrease in urinary epinephrine, norepinephrine, metanephrine and normetanephrine:creatinine ratios due to the cortisol-lowering effect of trilostane.

\section{Methods}

\section{Animals}

Fourteen dogs with naturally occurring hypercortisolism (HC) were prospectively enrolled. Results of catecholamines and metanephrines:creatinine ratios before trilostane therapy of five dogs had been previously reported as part of a former study [10]. Nine dogs were male (4 castrated) and five dogs female (4 spayed). Breeds included Dachshund (1), Giant Schnauzer (1), Nova Scotia Duck Tolling Retriever (1), Parson Jack Russell Terrier (1), Petit Bleu de Gascogne (1), Tibetan Terrier (1), Yorkshire Terrier (1), and 7 mixed-breed dogs. Age ranged between 6 and 14 years (median 8.5; standard error of mean (SEM): 0.6 ) and body weight between 7 and $58 \mathrm{~kg}$ (median 18.8; SEM: 3.7). Criteria for inclusion were the presence of clinical signs consistent with $\mathrm{HC}$ (e.g. polyuria, polydipsia, polyphagia, panting, skin signs, weakness, abdominal enlargement), a low-dose dexamethasone suppression test (LDDS test) or ACTH stimulation test results compatible with $\mathrm{HC}$ and the owner's agreement to treat and regularly re-evaluate the dog over at least a 6-month period [9, 11]. Pituitarydependent hypercortisolism (PDH) was diagnosed in all dogs by means of a normal or increased concentration of endogenous $\mathrm{ACTH}$, bilateral symmetrical appearance of the adrenal gland determined by ultrasonography and/or demonstration of pituitary enlargement by computed tomography.

Twenty-five client-owned dogs were enrolled as controls. These dogs had been part of previous studies [9, $10,12]$. Eleven dogs were male (6 castrated) and 14 female (12 spayed), and breeds included Australian Shepherd (1), Berger Blanc Suisse (1), Bernese Mountain Dogs (2), Border Collie (1), Golden Retriever (2), Gordon Setters (2), Labrador Retriever (2), Nova Scotia Duck Tolling Retriever (1), Rhodesian Ridgeback (1), Siberian Huskies (2), Standard Poodle (1), Tervueren (1), and eight mixed-breed dogs. Age ranged between 2 and 15 years (median 7; SEM: 0.6) and body weight between 10.4 and $59.2 \mathrm{~kg}$ (median 26; SEM: 2.3). The dogs were considered as healthy, based on detailed information provided by their owners and the results of a physical examination, $\mathrm{CBC}$, serum biochemistry profile, and urinalysis. The inclusion of the dogs in the study was approved by the veterinary office of the canton of Zurich and was in accordance with the guidelines and directives established by the Animal Welfare Act of Switzerland (TVB 199/2004).

\section{Sample collecting and processing}

All urine samples (healthy and sick dogs) used for analysis of catecholamines, metanephrines and creatinine were taken in hospital, either by free catch or by cystocentesis. In dogs with $\mathrm{HC}$ the first sample was taken in the morning during the initial work up for $\mathrm{HC}$ and once during trilostane therapy (at least 6 months after start of trilostane therapy). During trilostane therapy the urine was collected 2-3 $\mathrm{h}$ after the dose of trilostane in the morning. Urine collection and processing was done as reported previously $[9,12,13]$. Briefly, $10 \mathrm{ml}$ of urine were placed in a silicone-coated tube containing $270-280 \mu \mathrm{L}$ of $20 \%$ hydrochloric acid $(\mathrm{HCl})$. Urinary $\mathrm{pH}$ was measured using $\mathrm{pH}$ indicator strips (range of $\mathrm{pH} 1-6$ ) and $\mathrm{HCl}$ was added to achieve a $\mathrm{pH} \leq 2$ if needed. Samples were light protected and stored at $-20^{\circ} \mathrm{C}$ until analysis.

\section{Measurement of Catecholamines and Metanephrines}

Urine samples were analysed at the Institute of Clinical Chemistry, University Hospital Zurich, Zurich, Switzerland as previously described $[9,12,13]$. Urinary epinephrine, norepinephrine, total metanephrine and total normetanephrine were quantified by High Pressure Liquid Chromatography with amperometric detection as separate compounds. The terms "catecholamines" or "metanephrines" (plural form) includes epinephrine and 
norepinephrine or normetanephrine and metanephrine, respectively. The results were expressed as a ratio to urinary creatinine concentrations and will be listed in the following sections as: norepinephrine:creatinine, epinephrine:creatinine, normetanephrine:creatinine and metanephrine:creatinine.

\section{Measurement of cortisol and endogenous ACTH}

For the ACTH stimulation test, blood samples were taken before, and $60 \mathrm{~min}$ after, intravenous injection of $5 \mu \mathrm{g} / \mathrm{kg}$ synthetic tetracosactide (Synacthen ${ }^{\circ}$, Novartis Pharma Schweiz AG, Bern, Switzerland). Cortisol concentrations were measured by chemiluminescence assay (DPC Immulite 1000, Siemens Schweiz AG, Zurich, Switzerland). The intra-assay coefficients of variation were $10 \%$ and $6.3 \%$ at cortisol levels of 74.5 and $521 \mathrm{nmol} / \mathrm{L}$, respectively. The sensitivity of the assay was $5.5 \mathrm{nmol} / \mathrm{L}$. Endogenous ACTH before ACTH stimulation was determined by a chemiluminescence assay (DPC Immulite 1000, Siemens Schweiz AG, Zurich, Switzerland) previously validated for dogs [14, 15]. Blood was collected into chilled EDTA-coated tubes placed on ice and centrifuged at $4{ }^{\circ} \mathrm{C}$. Cortisol and endogenous $\mathrm{ACTH}$ measurements were performed in house twice a week; plasma was stored either at $-20{ }^{\circ} \mathrm{C}$ (cortisol) or at $-80^{\circ} \mathrm{C}(\mathrm{ACTH})$ until assayed.

\section{Experimental design}

The prospective study was performed between May 2006 and October 2014 at our hospital. The initial dose of trilostane for dogs with $\mathrm{HC}$ was $1-2 \mathrm{mg} / \mathrm{kg} \mathrm{q} 24 \mathrm{~h}$ (in the morning). ACTH stimulation tests were performed prior to trilostane treatment (except for $1 \mathrm{dog}$ ) and at regular intervals thereafter $(2,4,8,12$ and 16 weeks). The test was performed 2-3 $\mathrm{h}$ after the daily dose of trilostane according to the previously described treatment protocol [16]. The treatment goal was to achieve a postACTH cortisol concentration of 41-138 nmol/L. The dose of trilostane was adjusted in dogs with post-ACTH serum cortisol $>138 \mathrm{nmol} / \mathrm{L}$ or $<41 \mathrm{nmol} / \mathrm{L}$ and clinical signs suggestive of hypercortisolism (polyuria, polydipsia, polyphagia) or hypocortisolism (reduced appetite, vomiting or nausea, soft feces), respectively.

Urine samples for determination of catecholamines and metanephrines were collected at diagnosis of $\mathrm{HC}$ and at least 6 months after starting trilostane therapy (ensuring that all dogs were clinically controlled for at least 2 months). At the re-evaluation during trilostane therapy, dogs had to be clinically controlled (polyuria, polydipsia and panting decreased, weakness and agility increased), owners had to be satisfied with the treatment outcome and post-ACTH cortisol concentrations had to be $<138 \mathrm{nmol} / \mathrm{L}$. Median time for urinary sample collection during trilostane therapy was 11 months (range: 6-40 months) after diagnosis.

\section{Statistical analysis}

Data were analyzed with non-parametric statistical methods (GraphPad Prism6, Graph Pad Software, San Diego, CA, USA, SPSS 22.0 for Windows, SPSS Inc., Chicago, IL USA). Ranges and median values are reported. The Wilcoxon signed rank test was used for comparisons between different time points, and the Mann-Whitney U-Test for comparisons between different groups. Linear correlations were calculated by Spearman's non-parametric correlation. Values of $p<0.05$ were considered significant.

Preliminary reference intervals for the urinary catecholamines and metanephrines were generated by the nonparametric method of percentile estimates with confidence intervals to determine the central 95th percentile interval (i.e. 2.5 through 97.5 th percentile range) for results from clinically normal dogs [17]. Reference intervals thus determined were: epinephrine: creatinine $=1-18$; norepinephrine: creatinine $=1-19$; metanephrine: creatinine $=12$ 255; normetanephrine: creatinine $=14-123$.

\section{Results \\ Catecholamines and metanephrines before and during therapy}

Concentrations of catecholamines and metanephrines for dogs with $\mathrm{HC}$ before and during trilostane therapy and for healthy dogs are depicted in Fig. 1 and Table 1. In one dog the metanephrine:creatinine ratio could not be measured during trilostane therapy due to a chemical artefact.

The epinephrine, norepinephrine, and normetanephrine:creatinine ratios before trilostane therapy and the norepinephrine and normetanephrine:creatinine ratios during trilostane therapy of dogs with $\mathrm{HC}$ were significantly higher than those of healthy dogs (before: $p<0.01,<0.01,<0.0001$, respectively; during: $p<0.01$, $<0.001$, respectively) (Fig. 1).

There was no significant decrease in the epinephrine, norepinephrine, metanephrine and normetanephrine:creatinine ratios of dogs with $\mathrm{HC}$ during trilostane therapy ( $p=0.1,0.8,0.2,1$, respectively) (Fig. 1 ).

Grouping dogs according to their post-ACTH cortisol concentration during trilostane therapy, revealed 9 dogs with post-ACTH cortisol between 41 and $138 \mathrm{nmol} / \mathrm{L}$ (group 1) and 5 with post-ACTH cortisol $<41 \mathrm{nmol} / \mathrm{L}$ (group 2, no clinical signs of hypocortisolism evident). Concentrations of catecholamines and metanephrines for both groups during trilostane therapy and for healthy dogs are depicted in Fig. 2 and Table 2.

There was no significant difference in the epinephrine, norepinephrine, metanephrine and normetanephrine:creatinine ratios between the two groups during therapy ( $p=0.3,0.6,0.6,0.3$, respectively) (Fig. 2). Likewise 

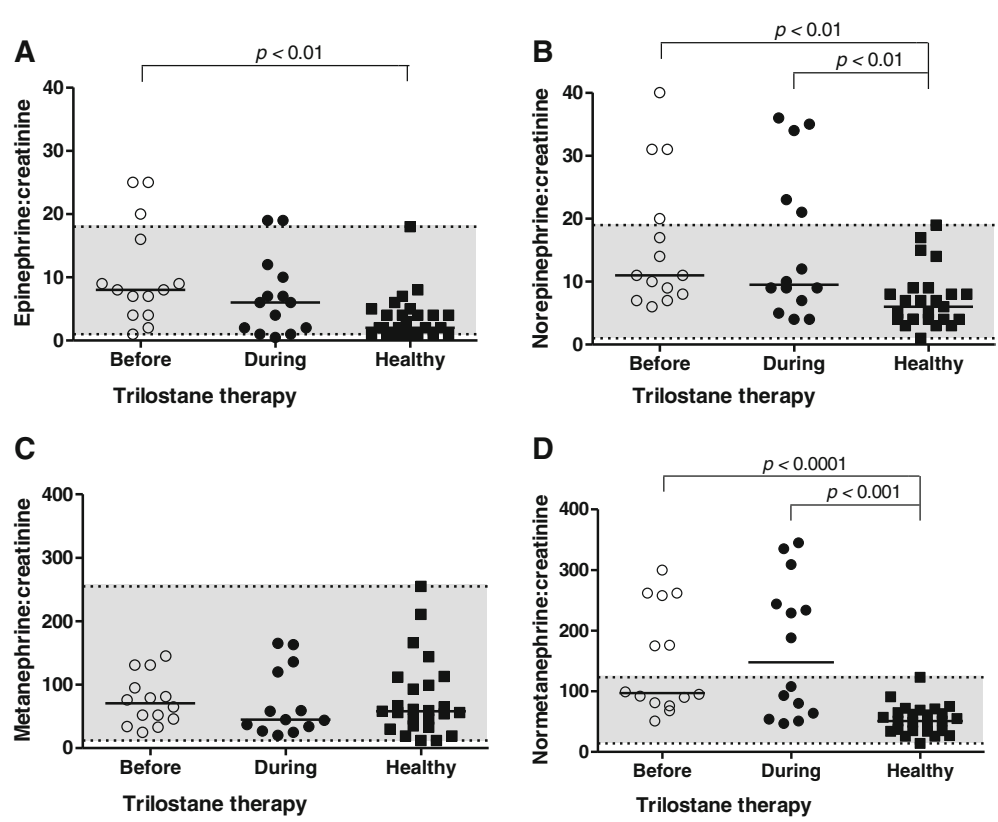

Fig. 1 Urinary epinephrine (a), norepinephrine (b), metanephrine (c) and normetanephrine:creatinine (d) in dogs with hypercortisolism before (open circles) and during (closed circles) trilostane therapy and in healthy dogs (squares). The horizontal bars represent the median of each group. The shaded area indicates the preliminary reference interval

epinephrine, norepinephrine, metanephrine and normetanephrine:creatinine ratios did not decrease significantly during treatment in both groups (group 1: $p=0.3,0.7$, $0.5,0.6$, respectively; group $2: p=0.6,0.5,0.6,0.6$, respectively). Compared to healthy dogs, however, dogs with post-ACTH cortisol between 41 and $138 \mathrm{nmol} / \mathrm{L}$ still had significantly increased epinephrine, norepinephrine and normetanephrine:creatinine ratios $(p<0.05$, $<0.01,<0.001$, respectively), whereas in dogs with post-ACTH cortisol $<41 \mathrm{nmol} / \mathrm{L}$ these differences were diminished ( $p=0.9,0.2,0.07$, respectively) (Fig. 2).

There was no significant correlation of epinephrine, norepinephrine, metanephrine or normetanephrine:creatinine ratios with baseline or post-ACTH-stimulated cortisol or

Table 1 Ranges (median/SEM) of urinary catecholamines and metanephrines to creatinine ratios in dog with hypercortisolism ( $\mathrm{HC}$ ) before and during trilostane therapy and in healthy dogs

\begin{tabular}{llll}
\hline Parameters & \multicolumn{2}{l}{ Dogs with HC } & Healthy dogs \\
\cline { 2 - 3 } & before therapy & during therapy & \\
\hline Epinephrine: & $1-25^{\mathrm{a}}$ & $0.5-19$ & $1-18$ \\
creatinine & $(8 / 2.1)$ & $(6 / 1.7)$ & $(2 / 0.7)$ \\
Norepinephrine: & $6-40^{\mathrm{a}}$ & $4-36^{\mathrm{a}}$ & $1-19$ \\
creatinine & $(11 / 2.9)$ & $(9.5 / 3.2)$ & $(6 / 0.9)$ \\
Metanephrine: & $25-145$ & $20-165$ & $12-255$ \\
creatinine & $(70.5 / 10.4)$ & $(45 / 14.9)$ & $(58 / 12.3)$ \\
Normetanephrine: & $51-300^{\mathrm{a}}$ & $47-345^{\mathrm{a}}$ & $14-123$ \\
creatinine & $(97 / 23.4)$ & $(148 / 29.9)$ & $(51 / 4.8)$ \\
\hline
\end{tabular}

${ }^{a}$ significant difference compared to healthy dogs with endogenous $\mathrm{ACTH}$ concentrations $(n=6)$ during trilostane therapy (Table 3).

\section{Discussion}

As expected, some dogs with $\mathrm{HC}$ had increased urinary catecholamines and metanephrines. This is in agreement with results from previous studies, which showed that in up to $50 \%$ of dogs with HC urinary epinephrine, norepinephrine and normetanephrine:creatinine ratios are above those of control dogs $[9,10]$. These findings most likely reflect the increased catecholamines synthesis (epinephrine and norepinephrine) and metabolism (conversion into metanephrine and normetanephrine) induced by glucocorticoids [3-7].

Unexpectedly, urinary catecholamines and metanephrines did not decrease significantly during trilostane therapy in dogs with $\mathrm{HC}$, and thus contradicted our hypothesis. There are several possible explanations for this finding. After once-daily trilostane administration cortisol concentrations are unlikely to be reduced over a 24-h period. Trilostane is an orally administered competitive inhibitor of the $3 \beta$-hydroxysteroid dehydrogenase, which mediates the conversion of pregnenolone to progesterone in the adrenal glands [18]. Cortisol, aldosterone and androstenedione are produced from progesterone via different biochemical pathways. Trilostane inhibits the production of progesterone and therefore the synthesis of its end products [19]. It is known that the maximum inhibitory effect of trilostane on glucocorticoid production in dogs is reached 2-4 $\mathrm{h}$ after its administration, and that cortisol concentrations re-increase thereafter [20]. In the 

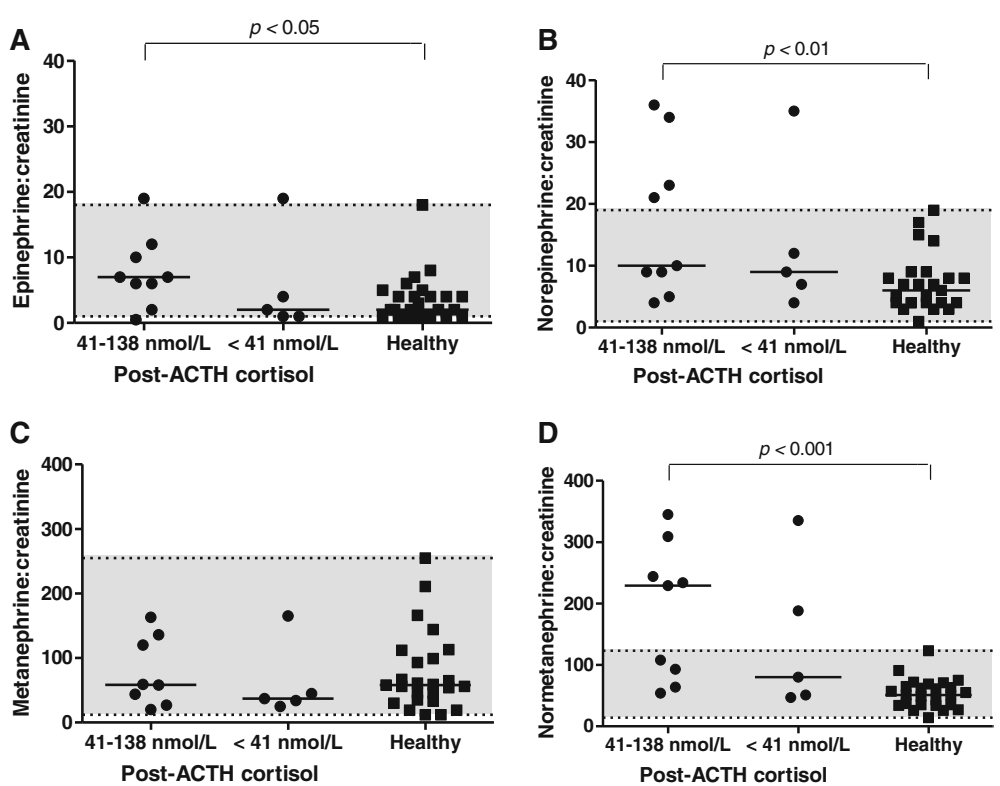

Fig. 2 Urinary epinephrine (a), norepinephrine (b), metanephrine (c) and normetanephrine:creatinine (d) in dogs with hypercortisolism during trilostane therapy according to their post-ACTH cortisol concentration (41-138 nmol/L or $<41 \mathrm{nmol} / \mathrm{L})$. The horizontal bars represent the median of each group. The shaded area indicates the preliminary reference interval

present study trilostane was dosed once-daily. Twice-daily therapy would lead to a more pronounced decrease in catecholamines and metanephrines concentrations than once-daily therapy, but this hypothesis has to be determined in a future study. Supporting evidence for this hypothesis is that dogs with low post-ACTH cortisol concentration $(<41 \mathrm{nmol} / \mathrm{L})$ had a diminished increase in epinephrine, norepinephrine and normetanephrine:creatinine ratios compared to healthy dogs. In contrast, the increase in epinephrine, norepinephrine and normetanephrine:creatinine ratios persisted in the dogs with post-ACTH cortisol between 41 and $138 \mathrm{nmol} / \mathrm{L}$.

Cortisol secretion during trilostane treatment has been evaluated in a study by Galac et al., where the authors

Table 2 Ranges (median/SEM) of urinary catecholamines and metanephrines to creatinine ratios in dog during trilostane therapy with post-ACTH cortisol between 41 and $138 \mathrm{nmol} / \mathrm{L}$ (group 1) and $<41 \mathrm{nmol} / \mathrm{L}$ (group 2) and in healthy dogs

\begin{tabular}{llll}
\hline Parameters & \multicolumn{2}{l}{ Dogs with HC } & Healthy dogs \\
\cline { 2 - 3 } & Group 1 & Group 2 & \\
\hline Epinephrine:creatinine & $0.5-19^{\mathrm{a}}$ & $1-19$ & $1-18$ \\
& $(7 / 1.8)$ & $(2 / 3.4)$ & $(2 / 0.7)$ \\
Norepinephrine:creatinine & $4-36^{\mathrm{a}}$ & $4-35$ & $1-19$ \\
& $(10 / 4.1)$ & $(9 / 5.6)$ & $(6 / 0.9)$ \\
Metanephrine:creatinine & $20-163$ & $25-165$ & $12-255$ \\
& $(59 / 19)$ & $(37 / 26.2)$ & $(58 / 12.3)$ \\
Normetanephrine:creatinine & $54-345^{\mathrm{a}}$ & $47-335$ & $14-123$ \\
& $(229 / 36.3)$ & $(80 / 55)$ & $(51 / 4.8)$ \\
\hline
\end{tabular}

${ }^{a}$ significant difference compared to healthy dogs compared urinary corticoid to creatinine ratios (UCCRs) in dogs with PDH before and during trilostane treatment after several months of optimal dosing of trilostane [21]. In that study, although UCCRs decreased significantly during therapy, they did not decrease below the upper limit of the reference range in the majority of the dogs, which might suggest that the influence of cortisol on the body system in trilostane-treated dogs persists, even in controlled dogs. Another interesting result in their study was that during long-term follow-up, a decrease in the UCCR below the upper limit of the reference range was associated with hypocortisolism. This seems similar to our results, where, as mentioned above, only dogs with a post-ACTH cortisol $<41 \mathrm{nmol} / \mathrm{L}$ had urinary

Table 3 Correlations between urinary catecholamines and metanephrines with cortisol and endogenous ACTH concentrations during trilostane therapy

\begin{tabular}{llll}
\hline Parameter & \multicolumn{3}{l}{ Spearman correlation coefficient / p-value } \\
\cline { 2 - 4 } & $\begin{array}{l}\text { Baseline } \\
\text { cortisol }\end{array}$ & $\begin{array}{l}\text { Post-ACTH } \\
\text { cortisol }\end{array}$ & Endogenous ACTH \\
\hline $\begin{array}{l}\text { Epinephrine: } \\
\text { creatinine }\end{array}$ & $0.01 / 1$ & $0.5 / 0.05$ & $0.2 / 0.7$ \\
$\begin{array}{l}\text { Norepinephrine: } \\
\text { creatinine }\end{array}$ & $-0.18 / 0.5$ & $0.4 / 0.2$ & $-0.1 / 0.8$ \\
$\begin{array}{l}\text { Metanephrine: } \\
\text { creatinine }\end{array}$ & $0.06 / 0.9$ & $0.5 / 0.1$ & $-0.2 / 0.7$ \\
$\begin{array}{l}\text { Normetanephrine: } \\
\text { creatinine }\end{array}$ & $0.16 / 0.6$ & $0.4 / 0.2$ & $-0.8 / 0.06$ \\
\hline
\end{tabular}


catecholamines and metenaphrines comparable to those of healthy dogs.

Another explanation for the failure to demonstrate a reduction in urinary catecholamines and metanephrines during trilostane therapy might be related to the blood supply of the adrenal medulla. The adrenal medulla gets its main blood supply through the capillaries of the cortex via the cortical-medullary portal system [1]. Due to this vascular system, the adrenal medulla gets in contact with glucocorticoids. In dogs with $\mathrm{HC}$, even though controlled during trilostane therapy, the glucocorticoid concentration, which reaches the adrenal medulla through the portal system, is probably still much higher than under physiologic conditions.

Plasma free catecholamines and metanephrines or urinary catecholamines and metanephrines:creatinine ratios are used to diagnose canine pheochromocytomas [22]. Clinical signs usually result from secretion of excessive amounts of catecholamines, and only rarely from the space-occupying or invasive nature of the tumor [23-29]. Hypercortisolism is one of the most important differential diagnoses of pheochromocytoma. Both diseases may have similar clinical signs (weakness, polyuria, polydipsia, panting) as well as similar ultrasonographic features; in rare cases they can even occur concurrently [10]. In dogs, measurement of plasma free normetanephrine and urinary normetanephrine:creatinine ratio have been shown to be the most reliable biochemical test for diagnosing pheochromocytoma, even in cases in which the two diseases occur concurrently $[9,10,13,30]$. Metanephrine to creatinine ratios were also significantly higher in dogs with pheochromocytoma compared to those of dogs with HC, but a considerable overlap existed [10]. This is similar to results described in human medicine, where normetanephrine concentrations have the highest sensitivity and specificity in diagnosing pheochromocytoma [31, 32]. Most likely, this reflects the fact that the vast majority of human and canine pheochromocytomas predominantly produce norepinephrine, which is metabolized to normetanephrine [10, 30, 31, 33]. Our study shows that dogs with $\mathrm{HC}$ treated with once-daily trilostane still have increased urinary concentrations of catecholamines and metanephrines and that diagnosing pheochromocytoma in these dogs might be challenging.

A limitation of the study is its rather low sample size. Another potential limitation is the lack of histopathology to exclude concurrent pheochromocytoma. However, only post mortem histopathology would reliably exclude concurrent pheochromocytoma, which is difficult to obtain in a clinical setting with medically treated patients. To minimize the risk of concurrent pheochromocytoma, we included only dogs with no obvious adrenal mass and with good clinical control after 6 months of trilostane therapy. Even though all dogs included seemed clinically well controlled, some dogs might have been overdosed according to their post-ACTH cortisol value $(<41 \mathrm{nmol} / \mathrm{L})$. This lack of a proven gold standard test is another limitation of studies evaluating dogs during trilostane therapy.

\section{Conclusions}

Although dogs with $\mathrm{HC}$ can be controlled during oncedaily trilostane therapy, it is likely that urinary concentrations of catecholamines and metanephrines remain increased, reflecting a constant influence of the endogenously increased cortisol concentration in these dogs.

\section{Abbreviations \\ HC: hypercortisolism; HCl: hydrochloric acid; LDDS test: low-dose dexamethasone suppression test; L-DOPA: L-dihydroxyphenylalanine: PDH: pituitary-dependent hyperadrenocorticism; PNMT: phenylethanolamin- $\mathrm{N}$-Methyltransferase; UCCRs: urinary corticoid to creatinine ratio}

\section{Acknowledgments \\ The authors gratefully acknowledge the veterinarians of the Clinic for Small Animal Internal Medicine for their contribution of cases. We thank all dog owners for their assistance and willingness to take part in this study.}

\section{Funding \\ This research received no grant from any funding agency in the public, commercial or not-for-profit sectors.}

\section{Availability of data and materials}

All data supporting the findings of the present study are included within the manuscript.

\section{Authors' contributions}

$N S R, F B, C R, E S, S Q$ participated in the conceptual aspect and design of the study. ES, SQ, NSR, FB, CR, KR were involved in the samples and data collection. ES, SQ, BR, KR, RHL performed the samples analysis. NSR, FB, CR, $\mathrm{BR}, \mathrm{RHL}, \mathrm{KR}$ performed statistical evaluation and data interpretation. NSR and FB wrote the first draft of the manuscript, with all authors involved in reviewing. All authors read and approved the final version of the manuscript.

\section{Ethics approval}

The use of the dogs was approved by the veterinary office of the canton of Zurich and was in accordance with the guidelines and directives established by the Animal Welfare Act of Switzerland (TVB 199/2004). Informed consent of all pet owners was obtained before including the dogs in the study.

Consent for publication

Not applicable.

\section{Competing interests}

The authors declare that they have no competing interests.

\section{Publisher's Note}

Springer Nature remains neutral with regard to jurisdictional claims in published maps and institutional affiliations.

\section{Author details}

${ }^{1}$ Clinic for Small Animal Internal Medicine, Vetsuisse Faculty University of Zurich, Zurich, Switzerland. ${ }^{2}$ Clinical Laboratory, Vetsuisse Faculty University of Zurich, Zurich, Switzerland. Institute of Clinical Chemistry, University Hospital Zurich, Zurich, Switzerland. ${ }^{4}$ Dr. Quante's current address is Peace Avenue Veterinary Clinic G/F, Hong Kong, China. 
Received: 20 January 2017 Accepted: 10 August 2017

\section{Published online: 04 September 2017}

\section{References}

1. Rosol TJ, Yarrington JT, Latendresse J, Capen CC. Adrenal gland: structure, function, and mechanisms of toxicity. Tox Pathol. 2001;29:41-18.

2. Eisenhofer G, Kopin IJ, Goldstein DS. Catecholamine metabolism: a contemporary view with implications for physiology and medicine. Pharmacol Rev. 2004:56:331-49.

3. Goodman R, Edgar D, Thoenen H, Wechsler W, Herschman H. Glucocorticoid induction of tyrosine hydroxylase in a continuous cell line of rat pheochromocytoma. J Cell Biol. 1978;78:R1-7.

4. Tai TC, Claycomb R, Her S, Bloom AK, Wong DL. Glucocorticoid responsiveness of the rat phenylethanolamine $\mathrm{N}$-methyltransferase gene. Mol Pharmacol. 2002;61:1385-92.

5. Yoshida-Hiroi M, Bradbury MJ, Eisenhofer G, Hiroi N, Vale WW, Novotny GE, Hartwig HG, Scherbaum WA, et al. Chromaffin cell function and structure is impaired in corticotropin-releasing hormone receptor type 1-null mice. Mol Psychiatry. 2002;7:967-74.

6. Wurtman $\mathrm{RH}$. Control of epinephrine synthesis in the adrenal medulla by the adrenal cortex: hormonal specificity and dose-response curves. Endocrinol. 1966;79:608-14.

7. Weiner N, Blaschko H. Adrenal gland endocrinology. In: Sayers G, Smith AD, editors. Endocrinology: adrenal gland. Washington DC: American Physiology Society; 1975.

8. Critchley JA, Henderrson CG, Moffat LE, Ungar A, Waite J, West CP. Proceedings: the release of catecholamines from perfused canine adrenal glands by corticoglucocorticoids. J Physiol. 1976;254:30P-1P.

9. Quante S, Boretti FS, Kook PH, Mueller C, Schellenberg S, Zini E, SieberRuckstuhl NS, Reusch CE. Urinary catecholamine and metanephrine to creatinine ratios in dogs with hyperadrenocorticism or pheochromocytoma, and in healthy dogs. J Vet Intern Med. 2010;24:1093-7.

10. Salesov E, Boretti FS, Sieber-Ruckstuhl NS, Rentsch KM, Riond B, HofmannLehmann R, Kircher PR, Grouzmann E, et al. Urinary and plasma catecholamines and metanephrines in dogs with pheochromocytoma, hypercortisolism, nonadrenal disease and in healthy dogs. J Vet Intern Med. 2015:29:597-602.

11. Mueller C, Sieber-Ruckstuhl NS, Wenger M, Kaser-Hotz B, Reusch CE. Lowdose dexamethasone test with "inverse" results: a possible new pattern of cortisol response. Vet Rec. 2006;159:489-91.

12. Kook PH, Boretti FS, Hersberger M, Glaus TM, Reusch CE. Urinary catecholamine and metanephrine to creatinine ratios in healthy dogs at home and in a hospital environment and in 2 dogs with pheochromocytoma. J Vet Intern Med. 2007;21:388-93.

13. Kook PH, Grest P, Quante S, Boretti FS, Reusch CE. Urinary catecholamine and metadrenaline to creatinine ratios in dogs with a pheochromocytoma. Vet Rec. 2010;166:169-74.

14. Rodriguez Pineiro MI, Benchekroun G, de Fornel-Thibaud P, Maurey-Guenec C, Garnier F, Rosenberg D. Accuracy of an adrenocorticotropic hormone $(\mathrm{ACTH})$ immunoluminometric assay for differentiating $\mathrm{ACTH}$-dependent from ACTH-independent hyperadrenocorticism in dogs. J Vet Intern Med. 2009;23:850-5.

15. Scott-Moncrieff JC, Koshko MA, Brown JA, Hill K, Refsal KR. Validation of a chemiluminescent enzyme immunometric assay for plasma adrenocorticotropic hormone in the dog. Vet Clin Pathol. 2003;32:180-7.

16. Braun C, Boretti FS, Reusch CE, Sieber-Ruckstuhl NS. Comparison of two treatment regimes with trilostane in dogs with pituitary-dependent hyperadrenocorticism. Schweiz Arch Tierheilkd. 2013;155:551-8.

17. Reed AH, Henry RJ, Mason WB. Influence of statistical method used on the resulting estimate of normal range. Clin Chem. 1971(17):275-84.

18. Ruckstuhl NS, Nett CS, Reusch CE. Results of clinical examinations, laboratory tests and ultrasonography in dogs with pituitary-dependent hyperadrenocorticism treated with trilostane. Am J Vet Res. 2002;63:506-12.

19. Potts GO, Creange JE, Hardomg HR, Schane HP. Trilostane, an orally active inhibitor of steroid biosynthesis. Steroids. 1978;32:257-67.

20. Lehnert C, Neiger R. 24 hour hormone and electrolyte levels of dogs with pituitary-dependent hyperadrenocorticism treated with trilostane. Giessen: Inaugural-Dissertation; 2007.

21. Galac S, Buijtels JJ, Kooistra HS. Urinary corticoid: creatinine ratios in dogs with pituitary-dependent hypercortisolism during trilostane treatment. J Vet Intern Med. 2009;23:1214-9.
22. Pacak K, Eisenhofer $G$, Ahlman $H$, Bornstein SR, Gimenez-Roqueplo AP, Grossman AB, Kimura N, Mannelli M, et al. Pheochromocytoma: recommendations for clinical practice form the first international symposium. October 2005. Nat Clin Pract Endocrinol Metab. 2007;3:92-102.

23. Berzon JL. A metastatic pheochromocytoma causing progressive paraparesis in a dog. Vet Med Small Anim Clin. 1981;76:675-9.

24. Bouayad H, Feeney DA, Caywood DD, Hayden DW. Pheochromocytoma in dogs: 13 cases (1980-1985). J Am Vet Med Assoc. 1987;191:1610-5.

25. Gilson SD, Withrow SJ, Wheeler SL, Twedt DC. Pheochromocytoma in 50 dogs. J Vet Intern Med. 1994;8:228-32.

26. Barthez PY, Marks SL, Woo J, Feldman EC, Matteucci M. Pheochromocytoma in dogs: 61 cases (1984-1995). J Vet Intern Med. 1997;11:272-8.

27. Out G. Pheochromocytoma in dogs: a retrospective study of nine cases (1981-1987). Can Vet J. 1989;30:526-7.

28. Platt SR, Sheppard BJ, Graham J, Uhl EW, Meeks J, Clemmons RM. Pheochromocytoma in the vertebral canal of two dogs. J Am Anim Hosp Assoc. 1998;34:365-71.

29. Santamarina G, Espino L, Vila M, Lopez M, Aleman N, Suarez ML. Aortic thromboembolism and retroperitoneal hemorrhage associated with a pheochromocytoma in a dog. J Vet Intern Med. 2003;17:917-22.

30. Gostelow R, Bridger N, Syme HM. Plasma-free metanephrine and free normetanephrine measurement for the diagnosis of pheochromocytoma in dogs. J Vet Intern Med. 2013;27:83-90.

31. Unger N, Pitt C, Schmidt IL, Walz MK, Schmid KW, Philipp T, Mann K, Petersenn S. Diagnostic value of various biochemical parameters for the diagnosis of pheochromocytoma in patients with adrenal mass. Eur J Endocrinol. 2006;154:409-17.

32. Guller U, Turek J, Eubanks S, Delon ER, Oertli D, Feldman JM. Detecting pheochromocytoma. Defining the most sensitive test. Ann Surg. 2006;243:102-7.

33. Eisenhofer G, Siegert G, Kotzerke J, Bornstein SR, Pacak K. Current progress and future challenges in the biochemical diagnosis and treatment of pheochromocytomas and paraganliomas. Horm Metab Res. 2008;40:329-37.

\section{Submit your next manuscript to BioMed Central and we will help you at every step:}

- We accept pre-submission inquiries

- Our selector tool helps you to find the most relevant journal

- We provide round the clock customer support

- Convenient online submission

- Thorough peer review

- Inclusion in PubMed and all major indexing services

- Maximum visibility for your research

Submit your manuscript at www.biomedcentral.com/submit
Biomed Central 